\title{
Effects of Cilnidipine on Heart Rate and Uric Acid Metabolism in Patients With Essential Hypertension
}

\author{
Arijit Das ${ }^{\mathrm{a}, \mathrm{f}}$, Prakash Kumar ${ }^{\mathrm{b}}$, Abha Kumari ${ }^{\mathrm{a}}$, Satish Chandra ${ }^{\mathrm{a}}$, Manju Garic, \\ Nidhi Singh ${ }^{\mathrm{d}}$, Debleena Dey ${ }^{\mathrm{e}}$
}

\begin{abstract}
Background: The relation between hypertension and hyperuricemia has been established by epidemiological studies. Calcium channel blockers are one of the first-line drugs for newly diagnosed patients with essential hypertension. Cilnidipine is a new calcium channel blocker acting by blocking both L- and N-type calcium channels. The aim of this study was to compare the effectiveness of amlodipine and cilnidipine in patients with essential hypertension and their effects on heart rate and serum uric acid levels.
\end{abstract}

Methods: Out of 100 enrolled patients, 92 completed the study. They were randomly assigned to amlodipine $(\mathrm{N}=47)$ and cilnidipine $(\mathrm{N}=$ 45) groups. Cilnidipine was started at $10 \mathrm{mg}$ /day and then adjusted to $5-20 \mathrm{mg} /$ day, and amlodipine was started at $5 \mathrm{mg} /$ day and then adjusted to $2.5-10 \mathrm{mg} /$ day.

Results: After 24 weeks of study, patients in cilnidipine groups showed significant reduction in heart rate and serum uric acid levels from baseline $(\mathrm{P}=0.00)$.

Conclusion: In clinical setting where both hypertension and hyperuricemia exist, cilnidipine can be a promising drug of choice.

Keywords: Hypertension; Heart rate; Hyperuricemia; Serum uric acid, L- and N-type calcium channel blocker; Cilnidipine

\section{Introduction}

Hypertension (HTN) is a chronic medical condition with per-

Manuscript accepted for publication August 23, 2016

a Department of Pharmacology, Rajendra Institute of Medical Sciences (RIMS), Ranchi, India

${ }^{b}$ Department of Cardiology, Rajendra Institute of Medical Sciences (RIMS), Ranchi, India

'Rajendra Institute of Medical Sciences (RIMS), Ranchi, India

${ }^{\mathrm{d} C}$ C.H. Hospital, Dhurwa, Ranchi, India

eIshpat Cooperative Hospital, Kolkata, India

${ }^{f}$ Corresponding Author: Arijit Das, Department of Pharmacology, Rajendra Institute of Medical Sciences (RIMS), Ranchi, India.

Email: 82.arijit@gmail.com

doi: http://dx.doi.org/10.14740/cr494w sistently elevated arterial blood pressure (BP) [1]. Around the globe, about 1 billion patients are affected by HTN and the burden is rising owing to escalating obesity and population aging and by 2025 it is projected that about 1.5 billion of patients will be affected worldwide [2]. Hyperuricemia affects $2-18 \%$ of the population with varying relation to age, sex, ethnicity and other epigenetic factor and can be regarded as a common metabolic disorder [3].

Many epidemiological studies confirm the relationship between the serum uric acid (SUA) level and various cardiovascular diseases, such as arterial HTN, acute and chronic heart failure, stroke, and atherosclerosis. Not only in patients with frank hyperuricemia (defined by SUA $>7 \mathrm{mg}$ in men and $>$ $6 \mathrm{mg}$ in women), but also in patients with high normal values of SUA (defined by SUA $>5.5 \mathrm{mg} / \mathrm{dL}$ ), similar relationship is evident $[4,5]$. It has been reported that $25-40 \%$ of patients with untreated HTN and more than $80 \%$ of patients with malignant HTN have high SUA levels [6].

The net balance between uric acid production and excretion is reflected as the SUA level. Renal insufficiency is a common cause of the increase in SUA and long-standing untreated hypertensive patients are at risk of developing chronic kidney disease (CKD). Hyperuricemia is highly prevalent in $\mathrm{CKD}$, reflecting the reducing renal excretion of SUA [6]. It has been long known that HTN is closely related to CKD and both conditions serve as risk factors for various cardiovascular diseases. Moreover, sympathetic hyperactivity in hypertensive patients and reduced nitric oxide production in the vascular endothelium caused by insulin resistance in hypertensive patients can lead to increased production of hypoxanthine in the skeletal muscle, resulting in so-called myogenic hyperuricemia [7].

Calcium channel blockers (CCBs) are one of the firstline recommended drugs for the treatment of HTN according to recent "Joint National Committee 8 guidelines" [8]. CCBs are divided structurally into the dihydropyridine type and the non-dihydropyridine type. Moreover, various subtypes of $\mathrm{Ca}^{2+}$ channels exist, such as L, N, T, P/Q, and R $[9,10]$.

The N-type voltage-dependent calcium channel plays an important role in sympathetic neurotransmission by regulating the release of norepinephrine from sympathetic nerve ending [11]. Cilnidipine is a novel and unique 1,4-dihydropyridine derivative calcium antagonist with potent inhibitory action against both L-type and N-type voltage-dependent calcium channels [12]. It has been reported that once daily administration of cilnidipine resulted in a safe and more effective lowering of BP in essential HTN without reflex tachycardia than 
similar administration of other dihydropyridine calcium antagonist [12]. In animal models, N-type CCBs not only suppress the sympathetic overactivity but also have cardioprotective and renoprotective effects. Renin-angiotensin-aldosterone system (RAAS) can be suppressed through the sympatholytic actions of N-type CCBs and thereby they may prevent impaired kidney function [13].

Currently various groups of antihypertensive drugs are available in the market and therefore selecting the appropriate drug for individual patient is of utmost importance in accordance with patient's clinical profile.

\section{Aim}

The aim of this study was to compare the effectiveness of amlodipine and cilnidipine in patients with essential HTN and their effects on heart rate and SUA levels.

\section{Materials and Methods}

\section{Study design}

This was an interventional, randomized, open-label, parallel assignment, comparative effectiveness study.

\section{Subjects}

We enrolled 100 patients with newly diagnosed essential HTN who presented to Outpatient Clinic of Cardiology, Rajendra Institute of Medical Sciences (RIMS), Ranchi, during January 2016. Patients were followed up for a period of 6 months. Patients were included in the study if they met the following criteria: $\geq 40$ years of age of both sex, systolic blood pressure (SBP)/diastolic blood pressure (DBP) of $\geq 140 / 90$ to $\leq$ $180 / 110 \mathrm{~mm} \mathrm{Hg}$. Patients with secondary HTN of any causes, angina pectoris or acute coronary artery disease, recent history of congestive heart failure, valvular heart diseases, cardiac arrhythmias, renal dysfunction (serum creatinine level $>$ $2.0 \mathrm{mg} / \mathrm{dL}$ ) and diabetes mellitus were excluded. Pregnant patients and breast feeding mothers were also excluded. Patients of any existing clinical condition which did not allow safe completion of the protocol and safe administration of study medication were also excluded. Target SBP and DBP were < 140 and $90 \mathrm{~mm} \mathrm{Hg}$, respectively. Eligible study subjects were randomly allocated to two groups and treated with cilnidipine (started at $10 \mathrm{mg} /$ day, and then adjusted to $5-20 \mathrm{mg} /$ day) or amlodipine (started at $5 \mathrm{mg} / \mathrm{day}$, and then adjusted to $2.5-10$ $\mathrm{mg} /$ day). If cilnidipine or amlodipine failed to reduce the BP to the target level, an angiotensin-converting-enzyme (ACE) inhibitor and/or angiotensin II receptor blockers (ARBs) were added and these patients were excluded from the study. Duration of follow-up was 6 months for each patient. Patients were followed on second, fourth, eighth, 12th and 24th week and at each visit in office, SBP, DBP and heart rate were recorded. BP was recorded three consecutive times and the average
Table 1. General Characteristics of Patients of Amlodipine and Cilnidipine Groups

\begin{tabular}{lll}
\hline Parameters & Amlodipine & Cilnidipine \\
\hline $\mathrm{N}$ & 47 & 45 \\
Male & 25 & 23 \\
Female & 22 & 22 \\
Age (years) & $52.86 \pm 5.81$ & $53.14 \pm 5.24$ \\
Body weight $(\mathrm{kg})$ & $67.56 \pm 10.85$ & $67.94 \pm 9.59$ \\
Height $(\mathrm{cm})$ & $162.22 \pm 6.79$ & $162.78 \pm 7.19$ \\
BMI & $25.01 \pm 5.1$ & $25.51 \pm 2.9$ \\
\hline
\end{tabular}

was taken as final value, using mercury sphygmomanometer. Blood samples were taken at baseline, 12th and 24th week between 9 am and 11 am and were immediately placed on ice and centrifuged within $1 \mathrm{~h}$ on first visit, 12th and 24th week. Uric acid was measured by an automatic biochemical analysis system.

\section{Ethics}

This study was conducted in accordance with the principles outlined in the Declaration of Helsinki after receiving approval from the institutional ethical committee of RIMS, Ranchi. All subjects provided written informed consent prior to participation.

\section{Statistical analysis}

The primary outcomes included changes in pulse rate, SBP, DBP and SUA in hypertensive patients following 24 weeks treatment with amlodipine or cilnidipine. Continuous data were presented as mean and standard deviation (SD). We compared values at baseline and after treatment using the "Z" test. All results were expressed as mean $\pm \mathrm{SD}$. P values $<0.05$ were considered significant. Statistical computations were performed with Microsoft Excel 2014 version.

\section{Results}

\section{Patient characteristics}

We analyzed the data of 48 men and 44 women with mean age of 53 years. Three patients of amlodipine group and five patients of cilnidipine group were lost in follow-up or excluded from the study due to side effects or inadequate control of BP with study medication by eighth week.

Table 1 summarizes the baseline characteristics of the patients enrolled for this study. There were no significant differences in background factors between the amlodipine and cilnidipine groups. 
Table 2. Comparison Data (SBP, DBP, mean BP, Heart Rate and Serum Uric Acid Level) of Baseline and After 24 Weeks of Treatment in Amlodipine and Cilnidipine Groups Along With Corresponding "Z" Test and P Values

\begin{tabular}{|c|c|c|c|c|c|c|c|c|}
\hline & \multicolumn{4}{|c|}{ Amlodipine } & \multicolumn{4}{|c|}{ Cilnidipine } \\
\hline & Baseline & 24th week & $\mathbf{Z}$ & $\mathbf{P}$ & Baseline & 24th week & $\mathbf{Z}$ & $\mathbf{P}$ \\
\hline SBP & $155.65 \pm 7.29$ & $133.38 \pm 6.39$ & 19.48 & $0.0^{*}$ & $155.38 \pm 6.76$ & $133.38 \pm 6.67$ & 19.23 & $0.00^{*}$ \\
\hline DBP & $93.32 \pm 3.97$ & $78.32 \pm 4.22$ & 18.29 & $0.00^{*}$ & $94.16 \pm 4.27$ & $79.92 \pm 4.27$ & 16.66 & $0.00^{*}$ \\
\hline Mean BP & $113.83 \pm 2.92$ & $96.45 \pm 3.52$ & 26.76 & $0.00^{*}$ & $114.48 \pm 2.64$ & $97.71 \pm 3.7$ & 26.09 & $0.00^{*}$ \\
\hline Heart rate & $76.88 \pm 4.37$ & $76.56 \pm 3.4$ & 0.40 & 0.68 & $76.96 \pm 4.53$ & $73 \pm 3.28$ & 4.99 & $0.00^{*}$ \\
\hline Uric acid & $5.67 \pm 1.31$ & $5.45 \pm 1.34$ & 0.88 & 0.37 & $5.94 \pm 1.09$ & $4.54 \pm 0.58$ & 4.28 & $0.00^{*}$ \\
\hline
\end{tabular}

*Significant.

Table 2 shows SBP, DBP and mean BP decreased significantly in both groups after treatment. There were no significant differences in the reduction in any of the BP parameters between amlodipine and cilnidipine groups. Heart rate reduced significantly from baseline in cilnidipine group after 24 weeks of treatment. SUA also shows significant reduction after 24 weeks of treatment in cilnidipine group.

\section{Discussion}

In the present study, both in amlodipine group and cilnidipine group, there was significant reduction in office BP readings and extent of reduction in $\mathrm{BP}$ is comparable in both the groups. $\mathrm{Al}-$ though changes in heart rate after 6 months of treatment from baseline were not significant in amlodipine group, the heart rate was significantly decreased in patients treated with cilnidipine (Fig. 1). Probable explanation for significant reduction of heart rate in cilnidipine group is due to the inhibition of cardiac sympathetic overactivity by blocking L- and N-type calcium channels [14]. Similar reduction of heart rate in cilnidipine group in comparison to amlodipine has been reported in other studies [11]. High heart rate is a known independent risk factor for cardiovascular death. Increased mortality from cardiovascular disease in Japanese population $[15,16]$ and in American [17] patients with HTN has been observed to be associated with a higher heart rate.

Reflex tachycardia is a common side effect associated with many dihydropyridine CCBs which act through L-type calcium channel only. Replacing these drugs with cilnidipine, a dual L- and N-type CCB may reduce the heart rate and can potentially reduce the mortality in hypertensive patients.

HTN and CKD are closely related and both serve as independent risk factors for various cardiovascular diseases. Hyperuricemia has also been reported to be involved in HTN and is a major risk factor in progression of CKD in addition to proteinuria $[18,19]$. Therefore, while treating HTN, it is desirable to use appropriate antihypertensives which have no adverse effect on uric acid metabolism.

In this study, we showed that after 24 weeks of treatment,

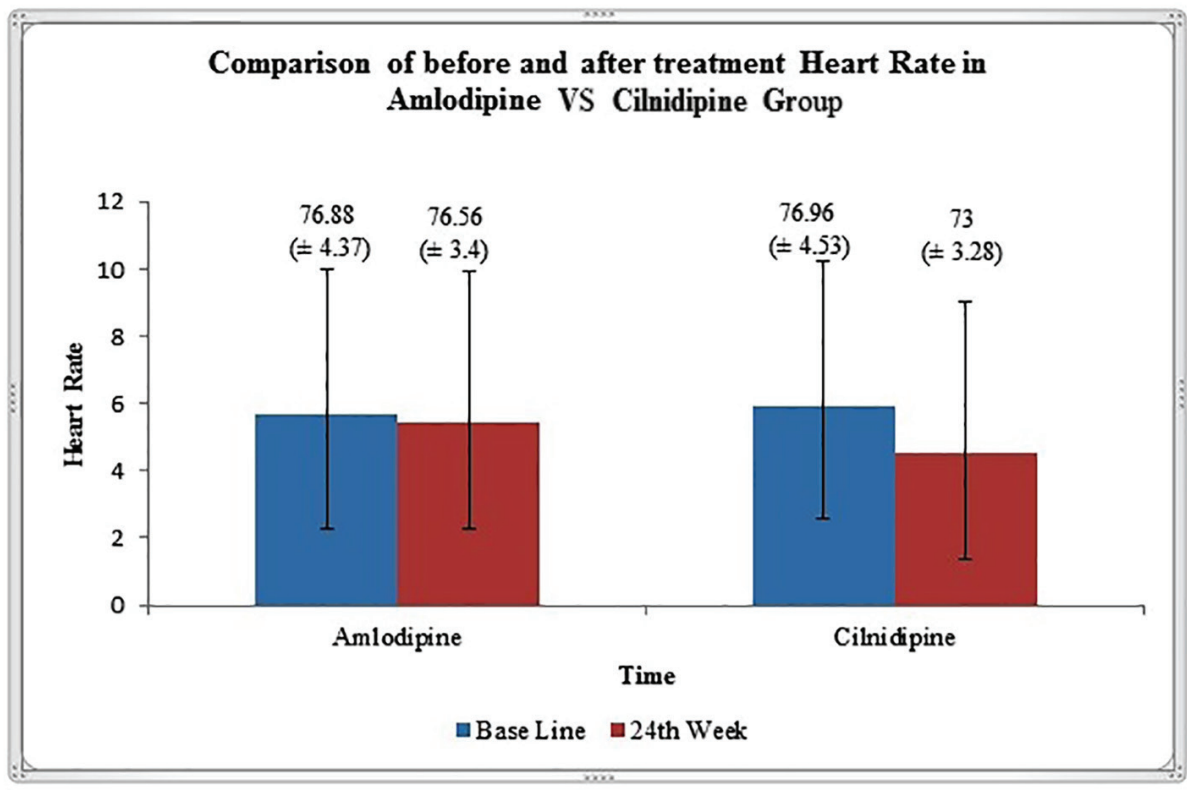

Figure 1. Graphical representation of heart rate (mean $\pm S D$ ) variation before and after 24 weeks of treatment in amlodipine and cilnidipine groups. 


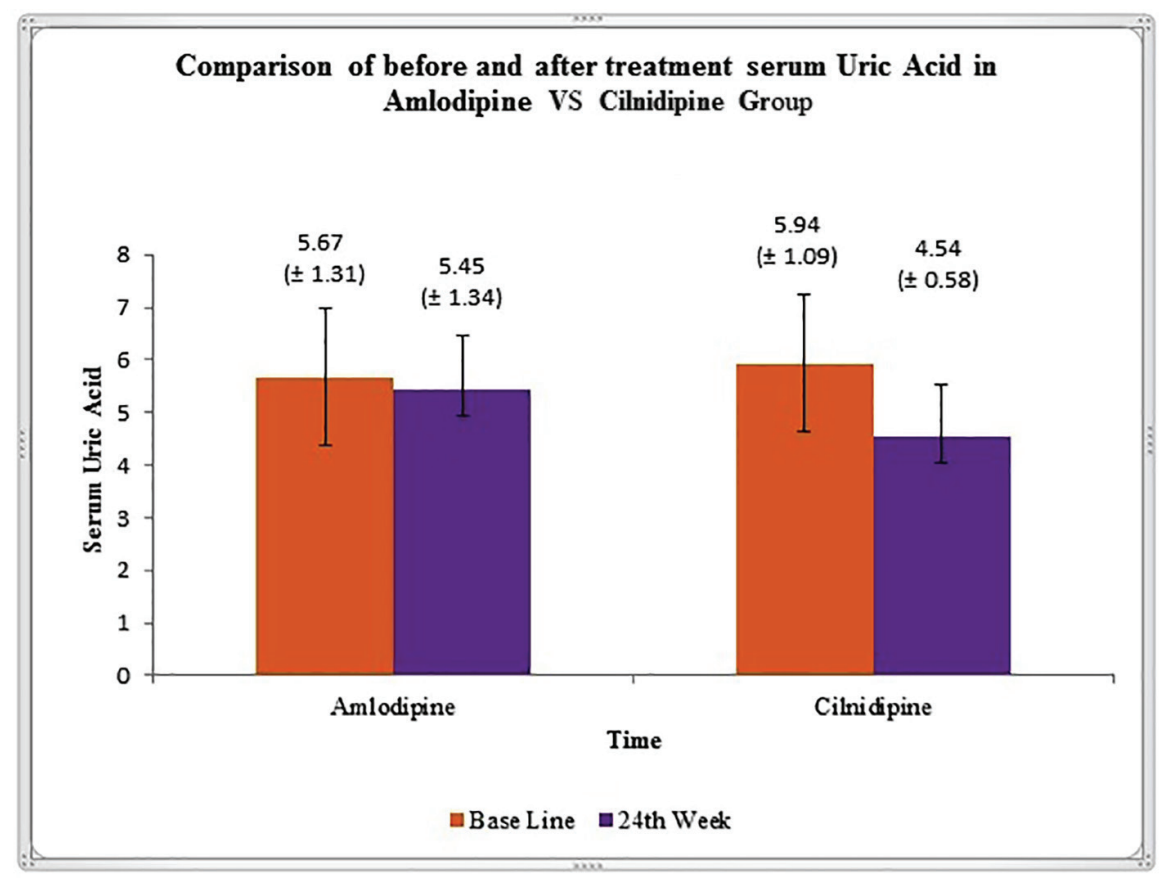

Figure 2. Graphical representation of variation in serum uric acid level (mean \pm SD), before and after 24 weeks of treatment in amlodipine and cilnidipine groups.

there is significant reduction in the level of SUA in cilnidipine group (Fig. 2). Studies by Hamada et al reported significant decrease in the SUA level and increased urinary nitrogen monoxide synthesis in hypertensive patients after treatment with cilnidipine [20]. Improvement of uric acid metabolism in hypertensive patients with CKD receiving cilnidipine has been observed in study by Uchida et al [7].

Animal study demonstrated suppression of hyperactivity of renal sympathetic nervous system by cilnidipine, which leads to intense dilatation of both afferent and efferent arterioles and a consequent reduction of glomerular filtration pressure [21]. Other studies showed suppression of renin, angiotensin, and aldosterone secretion by cilnidipine [22, 23]. In experimental study design using spontaneously hypertensive rats, cilnidipine showed suppression of RAAS activity and podocyte disorder [24]. Even clinically in the CARTER study, a trial involving switching from amlodipine to cilnidipine in patients with type 2 diabetes mellitus showed improvement in proteinuria by cilnidipine [25].

SUA reduction by cilnidipine may have several possible mechanisms. Firstly, suppression of sympathetic overactivity may suppress the uric acid precursor hypoxanthine formation. This is supported by evidence of suppression of sympathetic hyperactivity by $\alpha_{1}$ blockers and ACE inhibitors, leading to reduced production of hypoxanthine in the skeletal muscle, thus leading to improvement of myogenic hyperuricemia as seen in study by Ohtahara et al [26]. Secondly, cilnidipine by restoring blood flow to the skeletal muscle inhibits muscular AMP deaminase activity involved in ATP production, leading to suppressed degradation of AMP to hypoxanthine [7]. Improvement of renal blood flow due to afferent arteriolar dilatation [27] may lead to enhanced excretion of uric acid.
In the proximal convoluting tubules, a decrease in filtration fraction (i.e., glomerular filtration rate divided by renal plasma flow) eventually caused increased uric acid excretion [25, 28]. Lastly, the intestinal excretion of uric acid should also be considered. ABCG2 transporters mediate the intestinal excretion of uric acid. Single nucleotide polymorphisms (SNPs) of these transporters are found in substantial proportion gout patients [29]. In animal models in face of impaired kidney function, increased expression of ABCG2 transporters is observed as a compensatory mechanism to reduced urinary uric acid excretion [30].

Cilnidipine by virtue of its uric acid reducing property may provide better mortality protection in hypertensive patients.

\section{Limitations}

There are several limitations in this study. First, a small number of patients are enrolled in this study. Therefore, a large number of patients are needed to confirm our results. Second, the activity of RAAS and hypoxanthine was not evaluated in the present study. Third, further study on effect of cilnidipine on intestinal uric acid transporters is needed.

\section{Conclusion}

Taken together, the results from this study allow us to conclude that cilnidipine ( $\mathrm{N}$ - and L-type $\mathrm{CCB}$ ) exerts hypotensive activity, similar to the L-type CCB amlodipine; on the other hand, unlike amlodipine, it reduces heart rate and also improves uric acid metabolism. Considering these features, cilnidipine is a 
promising drug of choice for targeting patients with HTN and hyperuricemia, since both disorders often coexist in clinical setting.

\section{Grants}

The study was not supported by any grants or funding agencies.

\section{Conflicts of Interest}

The authors declare no conflicts of interest.

\section{References}

1. Naish Jeannette. Court, Denise Syndercombe. Medical sciences (2 ed.). 2014:562.

2. Lawes CM, Vander Hoorn S, Rodgers A. Global burden of blood-pressure-related disease, 2001. Lancet. 2008;371(9623):1513-1518.

3. Iliesiu A, Campeanu A, Dusceac D. Serum uric acid and cardiovascular disease. Maedica (Buchar). 2010;5(3):186192.

4. Niskanen LK, Laaksonen DE, Nyyssonen K, Alfthan G, Lakka HM, Lakka TA, Salonen JT. Uric acid level as a risk factor for cardiovascular and all-cause mortality in middle-aged men: a prospective cohort study. Arch Intern Med. 2004;164(14):1546-1551.

5. Feig DI, Kang DH, Johnson RJ. Uric acid and cardiovascular risk. N Engl J Med. 2008;359(17):1811-1821.

6. Iseki K, Oshiro S, Tozawa M, Iseki C, Ikemiya Y, Takishita S. Significance of hyperuricemia on the early detection of renal failure in a cohort of screened subjects. Hypertens Res. 2001;24(6):691-697.

7. Uchida S, Takahashi M, Sugawara M, Saito T, Nakai K, Fujita M, Mochizuki K, et al. Effects of the N/L-type calcium channel blocker cilnidipine on nephropathy and uric acid metabolism in hypertensive patients with chronic kidney disease (J-CIRCLE study). J Clin Hypertens (Greenwich). 2014;16(10):746-753.

8. James PA, Oparil S, Carter BL, Cushman WC, Dennison-Himmelfarb C, Handler J, Lackland DT, et al. 2014 evidence-based guideline for the management of high blood pressure in adults: report from the panel members appointed to the Eighth Joint National Committee (JNC 8). JAMA. 2014;311(5):507-520.

9. Fujii S, Kameyama K, Hosono M, Hayashi Y, Kitamura K. Effect of cilnidipine, a novel dihydropyridine $\mathrm{Ca}++$-channel antagonist, on $\mathrm{N}$-type $\mathrm{Ca}++$ channel in rat dorsal root ganglion neurons. J Pharmacol Exp Ther. 1997;280(3):1184-1191.

10. Hirning LD, Fox AP, McCleskey EW, Olivera BM, Thayer SA, Miller RJ, Tsien RW. Dominant role of N-type $\mathrm{Ca} 2+$ channels in evoked release of norepinephrine from sympathetic neurons. Science. 1988;239(4835):57-61.
11. Hoshide S, Kario K, Ishikawa J, Eguchi K, Shimada $\mathrm{K}$. Comparison of the effects of cilnidipine and amlodipine on ambulatory blood pressure. Hypertens Res. 2005;28(12):1003-1008.

12. Minami J, Ishimitsu T, Kawano Y, Numabe A, Matsuoka H. Comparison of 24-hour blood pressure, heart rate, and autonomic nerve activity in hypertensive patients treated with cilnidipine or nifedipine retard. J Cardiovasc Pharmacol. 1998;32(2):331-336.

13. Mori Y, Aritomi S, Niinuma K, Nakamura T, Matsuura K, Yokoyama J, Utsunomiya K. Additive effects of cilnidipine, an L-/N-type calcium channel blocker, and an angiotensin II receptor blocker on reducing cardiorenal damage in Otsuka Long-Evans Tokushima Fatty rats with type 2 diabetes mellitus. Drug Des Devel Ther. 2014;8:799-810.

14. Sakata K, Shirotani M, Yoshida H, Nawada R, Obayashi K, Togi K, Miho N. Effects of amlodipine and cilnidipine on cardiac sympathetic nervous system and neurohormonal status in essential hypertension. Hypertension. 1999;33(6):1447-1452.

15. Okamura T, Hayakawa T, Kadowaki T, Kita Y, Okayama A, Elliott P, Ueshima H. Resting heart rate and causespecific death in a 16.5-year cohort study of the Japanese general population. Am Heart J. 2004;147(6):1024-1032.

16. Hozawa A, Ohkubo T, Kikuya M, Ugajin T, Yamaguchi J, Asayama K, Metoki H, et al. Prognostic value of home heart rate for cardiovascular mortality in the general population: the Ohasama study. Am J Hypertens. 2004;17(11 Pt 1):1005-1010.

17. Gillman MW, Kannel WB, Belanger A, D'Agostino RB. Influence of heart rate on mortality among persons with hypertension: the Framingham Study. Am Heart J. 1993;125(4):1148-1154.

18. Heinig M, Johnson RJ. Role of uric acid in hypertension, renal disease, and metabolic syndrome. Cleve Clin J Med. 2006;73(12):1059-1064.

19. Krishnan E, Kwoh CK, Schumacher HR, Kuller L. Hyperuricemia and incidence of hypertension among men without metabolic syndrome. Hypertension. 2007;49(2):298303.

20. Hamada T, Yamada K, Mizuta E, Watanabe A, Osaki T, Ishida K, Hasegawa A, et al. Effects of cilnidipine on serum uric acid level and urinary nitrogen monoxide excretion in patients with hypertension. Clin Exp Hypertens. 2012;34(7):470-473.

21. Konno Y, Kimura K. Vasodilatory effect of cilnidipine, an L-type and N-type calcium channel blocker, on rat kidney glomerular arterioles. Int Heart J. 2008;49(6):723-732.

22. Konda T, Enomoto A, Aritomi S, Niinuma K, Koganei H, Ogawa T, Nitta K. Different effects of L/N-type and L-type calcium channel blockers on the renin-angiotensin-aldosterone system in SHR/Izm. Am J Nephrol. 2009;30(2):155-161.

23. Aritomi S, Wagatsuma $\mathrm{H}$, Numata T, Uriu $\mathrm{Y}$, Nogi $\mathrm{Y}$, Mitsui A, Konda T, et al. Expression of N-type calcium channels in human adrenocortical cells and their contribution to corticosteroid synthesis. Hypertens Res. 2011;34(2):193-201

24. Fan YY, Kohno M, Nakano D, Ohsaki H, Kobori H, Su- 
warni D, Ohashi N, et al. Cilnidipine suppresses podocyte injury and proteinuria in metabolic syndrome rats: possible involvement of N-type calcium channel in podocyte. J Hypertens. 2010;28(5):1034-1043.

25. Fujisawa T, Ikegami H, Noso S, Hiromine Y, Kawabata Y, Nishino M, Asano K, et al. Renoprotective effect of N-type Ca channel blockade in diabetic nephropathy. J Diabetes Complications. 2007;21(4):252-257.

26. Ohtahara A, Hisatome I, Yamamoto Y, Furuse M, Sonoyama K, Furuse Y, Hamada T, et al. The release of the substrate for xanthine oxidase in hypertensive patients was suppressed by angiotensin converting enzyme inhibitors and alpha1-blockers. J Hypertens. 2001;19(3 Pt 2):575582.

27. Zhou X, Ono H, Ono Y, Frohlich ED. N- and L-type calcium channel antagonist improves glomerular dynamics, reverses severe nephrosclerosis, and inhibits apoptosis and proliferation in an l-NAME/SHR model. J Hypertens. 2002;20(5):993-1000.

28. Fujita T, Ando K, Nishimura H, Ideura T, Yasuda G, Isshiki M, Takahashi K. Antiproteinuric effect of the calcium channel blocker cilnidipine added to renin-angiotensin inhibition in hypertensive patients with chronic renal disease. Kidney Int. 2007;72(12):1543-1549.

29. Ichida K, Matsuo H, Takada T, Nakayama A, Murakami K, Shimizu T, Yamanashi Y, et al. Decreased extra-renal urate excretion is a common cause of hyperuricemia. Nat Commun. 2012;3:764.

30. Yano H, Tamura Y, Kobayashi K, Tanemoto M, Uchida S. Uric acid transporter ABCG2 is increased in the intestine of the 5/6 nephrectomy rat model of chronic kidney disease. Clin Exp Nephrol. 2014;18(1):50-55. 